\title{
Novel chitosan-cellulose nanofiber self- healing hydrogels to correlate self-healing properties of hydrogels with neural regeneration effects
}

\author{
Kun-Chih Cheng ${ }^{1}$, Chih-Feng Huang ${ }^{2}$, Yen Wei ${ }^{3}$ and Shan-hui Hsu ${ }^{1,45}$
}

\begin{abstract}
Biodegradable self-healing hydrogels are attractive materials for tissue repair; however, the impact of the self-healing abilities of hydrogels on tissue repair is not clear. In this study, we prepared novel chitosan-cellulose nanofiber (CS-CNF) composite self-healing hydrogels with the same modulus (approximately $2 \mathrm{kPa}$ ) but tunable self-healing properties. By adding a low amount of CNFs (0.06-0.15 wt\%) in the pristine chitosan (CS) self-healing hydrogel, the reversible dynamic Schiff bonding, strain sensitivity, and self-healing of the hydrogel are obviously affected. Neural stem cells embedded in the CS-CNF hydrogel with better self-healing properties reveal significantly enhanced oxygen metabolism as well as neural differentiation. The differentiation of neural stem cells is highly correlated with their metabolic change in the self-healing hydrogel. Moreover, the neural regeneration effect of the optimized CS-CNF hydrogel with $0.09 \mathrm{wt} \%$ CNFs and the best self-healing properties show a 50\% improvement over the pristine CS hydrogel in the zebrafish brain injury model. A mechanism is proposed to interpret the tunable self-healing properties of CS-CNF hydrogels with stiffness maintained in a similar range. The new self-healing hydrogels help to clarify the role of self-healing in the biological performance of hydrogels as well as provide design rationale for hydrogels with better injectability and tissue regeneration potential.
\end{abstract}

\section{Introduction}

Hydrogel possesses the capability of holding a large amount of water in a three-dimensional (3D) network and is an attractive class of materials for biomedical applications ${ }^{1,2}$. Recently, stimulus-responsive hydrogels have attracted much attention because of their versatility and a broad range of applications. Hydrogels with self-healing properties provide appealing features such as the less short invasive delivery procedure by injection at the target site without gel fragmentation ${ }^{3}$. Self-healing hydrogels

Correspondence: Shan-hui Hsu (shhsu@ntu.edu.tw)

${ }^{1}$ Institute of Polymer Science and Engineering, National Taiwan University, Taipei, Taiwan, Republic of China

2Department of Chemical Engineering, National Chung Hsing University, Taichung, Taiwan, Republic of China

Full list of author information is available at the end of the article. with strong tissue adhesion significantly promote wound healing ${ }^{4,5}$. They may also carry therapeutic agents to the damaged tissue area, offering a local treatment effect. Moreover, the self-healing hydrogels may provide an extracellular matrix-like 3D environment for embedded cells and thus hold promises for tissue engineering applications ${ }^{6-8}$.

CS is a linear polysaccharide consisting of randomly distributed N-acetyl-D-glucosamine (acetylated) and $\beta$ - $(1 \rightarrow 4)$-linked D-glucosamine (deacetylated $)^{9,10}$, and is extracted by treating the chitin shells of shrimp and other crustaceans with an alkaline substance. CS has been widely applied in biomedical applications because of its good biocompatibility and biodegradability ${ }^{11-14}$. Wei and colleagues fabricated a biocompatible self-healing CSbased hydrogel as a new injectable cell therapy carrier ${ }^{15}$.

\section{(c) The Author(s) 2019}

(c) Open Access This article is licensed under a Creative Commons Attribution 4.0 International License, which permits use, sharing, adaptation, distribution and reproduction cc) in any medium or format, as long as you give appropriate credit to the original author(s) and the source, provide a link to the Creative Commons license, and indicate if changes were made. The images or other third party material in this article are included in the article's Creative Commons license, unless indicated otherwise in a credit line to the material. If material is not included in the article's Creative Commons license and your intended use is not permitted by statutory regulation or exceeds the permitted use, you will need to obtain permission directly from the copyright holder. To view a copy of this license, visit http://creativecommons.org/licenses/by/4.0/. 
Ding and colleagues fabricated CS-based hydrogels with multibenzaldehyde-functionalized poly(ethylene glycol) (PEG) analogs for cartilage tissue engineering ${ }^{7}$. Furthermore, CS-based hydrogel scaffolds immobilized with growth factors or containing endothelial cells were found to induce the differentiation of neural stem cells $(\mathrm{NSCs})^{16,17}$. When added with a small amount of fibrinogen, the CS self-healing hydrogel could form double network that maintained the self-healing property and acquired the ability to promote blood capillary formation ${ }^{18}$.

Cellulose is a linear polysaccharide containing several hundreds to many thousands of $\beta$ - $(1 \rightarrow 4)$-linked D-glucose, and is the most abundant organic polymer on earth $^{19,20}$. Natural fibrils of cellulose have a diameter of $2-20 \mathrm{~nm}$ and a crystallinity of $65-95 \%$, depending on their origins ${ }^{21,22}$. Cellulose nanofibers (CNFs) can be obtained from wood using 2,2,6,6-tetramethylpiperidinyl1-oxyl (TEMPO)-mediated oxidation, also named TEMPO-oxidized CNFs $(\mathrm{TOCNs})^{23-25}$. Through the process of partial carboxylation of the hydroxyl group, CNFs are extracted from native cellulose and uniformly dispersed in water. In addition to CNFs, nanocrystalline cellulose (NCC) and bacterial nanocellulose (BNC) have also been used in the biomedical field. For example, the hydrogel containing TEMPO-mediated oxidized BNC and alginate were employed to encapsulate the insulin secreting $\beta$-cells ${ }^{26}$.

CNFs obtained from the TEMPO-mediated oxidation of cellulose were used to prepare polymer nanocomposites with superior reinforcement effects ${ }^{24,27,28}$. Literature regarding the addition of CNFs in hydrogel is relatively rare. Very recently, CNFs (0.5-4.5 wt\%) were incorporated into methacrylate-functionalized carboxymethyl cellulose followed by UV crosslinking to generate mechanically robust hydrogels ${ }^{29}$. The fibrous structure of CNFs may presumably change the rheological behavior and modify the self-healing ability of the hydrogel network, which has not been explored thus far. In the current study, we prepared composite hydrogels from CS-based self-healing hydrogel and a very low amount of CNFs (0.06-0.15 wt\%). The self-healing, injectability, and rheological properties of the composite hydrogels were investigated. NSCs were embedded in the composite hydrogels and analyzed for cell proliferation, gene expression, protein expression, and aerobic metabolism. We demonstrated that the presence of CNFs modulated the self-healing behavior of composite hydrogels. In particular, the self-healing ability of the composite hydrogels was positively correlated with the neural differentiation of NSCs embedded in the hydrogel in vitro and the functional recovery of neural-impaired zebrafish in vivo.

\section{Materials and methods \\ Preparation of CNFs}

CNFs were prepared as previously described ${ }^{25}$. The CNF dispersion was obtained by mixing wood pulp, 2,2,6,6tetramethylpiperidine-1-oxyl (TEMPO), sodium bromide $(\mathrm{NaBr})$, and sodium hydroxide $(\mathrm{NaOH})$ in deionized water. Sodium hypochlorite $(\mathrm{NaClO})$ was then added to start the oxidation/reduction reactions. After $12 \mathrm{~h}$, the reaction was stopped by diluting with abundant deionized water. The dispersion was purified by repeated washing in deionized water until the aqueous solution reached neutral $\mathrm{pH}$, and was concentrated to obtain the gel-like CNFs and stored in a $4{ }^{\circ} \mathrm{C}$ refrigerator. The degree of substitution (i.e., extent of carboxylation) determined by titration (conductivity method) was approximately $30 \%$.

\section{Preparation of CS-CNF composite self-healing hydrogels}

Telechelic difunctional PEG (DF-PEG) was synthesized through esterification of hydroxyl-terminated PEG with 4formylbenzoic acid ${ }^{30}$. The solid powders of DF-PEG and glycol CS (molecular weight of $510 \mathrm{kDa}$, Sigma, St. Louis, MO, USA) were dissolved in deionized water. To obtain the composite hydrogels, the CNFs were first dispersed in DF-PEG before mixing with glycol CS. The gelation mechanism of the DF-PEG-crosslinked glycol CS hydrogel (chitosan-based hydrogel, abbreviated as CS hydrogel) was network formation through dynamic a Schiff base reaction ${ }^{16}$. The crosslinking density of the hydrogel was tuned by the amount of the crosslinker (DFPEG). The glycol CS and DF-PEG were dissolved in cell culture medium (described in Section "Three-dimensional (3D) cell culture in CS-CNF hydrogels"). The pristine CS hydrogel in this study was resulted from mixing an equal amount of solutions of glycol CS (3\%) and DF-PEG (2\%). Meanwhile, a series of composite CS hydrogels were prepared to contain different contents of CNFs $(0,0.06,0.09,0.12$, and $0.15 \mathrm{wt} \%)$ in the CS hydrogel. To obtain the composite hydrogels, the CNFs were first dispersed in DF-PEG before mixing with glycol CS.

\section{Rheological measurement of CS-CNF hydrogels}

The rheological properties of various hydrogels (CS and CS-CNF) were measured by a dynamic rheometer (HR-2, TA Instruments) with a cone $\left(40 \mathrm{~mm}\right.$ diameter with $2^{\circ}$ angle)-and-plate geometry. First, a 3\% glycol CS solution was prepared in culture medium and loaded onto the plate. Then, a $2 \%$ DF-PEG solution with different amounts of CNFs was added onto the glycol CS solution. The measurement was maintained at $37^{\circ} \mathrm{C}$. The storage modulus $\left(G^{\prime}\right)$ and loss modulus $\left(G^{\prime \prime}\right)$ were monitored at $1 \mathrm{~Hz}$ frequency and $1 \%$ oscillating shear strain for the 
time-dependent measurement. The strain sensitivity (the strain for the damage to occur) of various hydrogels was evaluated by conducting a strain sweep test at $1 \mathrm{~Hz}$ with the oscillatory shear strain increasing stepwise from 1 to $400 \%$. Based on the strain sweep results, the self-healing properties were evaluated by damage-healing cycles, which were continuous step changes in the oscillatory strain at $1 \mathrm{~Hz}$ between $1 \%$ and $n \%$ (where $n \%$ was the strain at which damage occurred measured from the strain sweep, depending on the content the of CNFs). The healing-damaging cycles (200 s each) provided information on the strain-induced destruction and recovery of the hydrogel.

\section{Thermal analyses and degradation studies of CS and CS-CNF hydrogels}

The thermal properties of the CS and CS-CNF hydrogels were evaluated by a thermogravimetric analyzer (TGA, Q50, TA Instrument, New Castle, DE, USA). The Dried samples $(4-8 \mathrm{mg})$ were heated from 100 to $800^{\circ} \mathrm{C}$ at a heating rate of $20^{\circ} \mathrm{C} / \mathrm{min}$ under a nitrogen atmosphere.

In addition, the biodegradation of the hydrogels was tested in vitro. Hydrogels weighing approximately $500 \mathrm{mg}$ were placed in $2 \mathrm{~mL}$ phosphate-buffered saline (PBS) solution (pH 7.4). The hydrogels were incubated at $37^{\circ} \mathrm{C}$ for $3,7,14,21$, and 28 days. The remaining mass (\%) was calculated from the equation: remaining mass $(\%)=\left(W_{\mathrm{t}}\right)$ $\left.W_{\mathrm{i}}\right) \times 100 \%$, where $W_{\mathrm{i}}$ is the initial weight of the hydrogel and $W_{\mathrm{t}}$ is the weight of the hydrogel at any time point.

\section{D cell culture in CS-CNF hydrogels}

NSCs were obtained from adult mouse brains as previously described ${ }^{31}$. Briefly, dissociated cells from the mouse brain were cultured in a cell culture dish (Corning, NY) with Dulbecco's modified Eagle's medium (DMEM, Gibco, USA) supplemented with $10 \%$ fetal bovine serum (FBS, Gibco, USA) in a $37^{\circ} \mathrm{C} / 5 \% \mathrm{CO}_{2}$ incubator. After cell colonies appeared in the dish, the serum-supplemented medium was switched to serum-free medium. The NSCs were then selected by $400 \mathrm{mg} / \mathrm{mL}$ G418 (Invitrogen) treatment as previously described ${ }^{31}$. The NSCs were maintained in Dulbecco's modified Eagle's medium and Ham's F-12 (DMEM/F12 (1:1), Gibco, USA) supplemented with $10 \%$ fetal bovine serum (FBS, Gibco,USA), $100 \mathrm{U} / \mathrm{mL}$ penicillin-streptomycin (PS, Caisson Labs, USA), and $400 \mathrm{mg} / \mathrm{mL}$ G418. The culture medium was refreshed once every 2 days.

In the 3D culture group, the NSCs and CNFs were suspended in DF-PEG containing NSC culture medium without G418 and were mixed with glycol CS dissolved in the same medium. The NSCs (cell density $2 \times 10^{6}$ per mL) embedded in the CS-CNF hydrogels were further cultured in NSC culture medium without G418.

\section{Cell viability and aerobic metabolism for cells in CS-CNF hydrogels}

The proliferation rate of cells was investigated in the long term by the CCK-8 (Sigma-Aldrich, Japan) assay. The NSCs (cell density $5 \times 10^{5}$ per $\mathrm{mL}$ ) were embedded in different hydrogels. At $0,3,7,14,21$, and 28 days, each hydrogel (containing NSCs) was washed and incubated with the CCK-8 reagent and culture medium at $37^{\circ} \mathrm{C}$ for $2 \mathrm{~h}$. The CCK-8 reagent entered the hydrogel in approximately two hours. After adding the CCK- 8 reagent, we incubated each set of experiments for the same amount of time. The supernatant of each hydrogel (containing NSCs) was then aspirated to a 96-well plate. Optical absorption was measured at $450 \mathrm{~nm}$ by a microplate reader (SpectraMax M5, Molecular Devices, USA), and the values were deducted from that of a blank control (pristine CS hydrogel without cells). The optical absorption values were deducted from that of a blank control (same hydrogel without cells) before the optical absorption values were normalized at day 0 . The values are expressed as the percentage (\%). Cell viability in the hydrogels was confirmed by confocal microscopy. NSCs (cell density $1 \times 10^{6}$ per $\mathrm{mL}$ ) embedded in the self-healing hydrogel were cultured for 3 days. 3D fluorescence images of the NSCs after acridine orange/4'6-diamidino-2-phenylindole (AO, green/DAPI, blue) staining by a confocal microscope (Leica TCS SP5 II) were obtained. Viability was calculated from the number of green spots divided by the sum of green and blue spots.

The oxygen metabolism and mitochondrial function of the cells in hydrogels were analyzed by the Seahorse XFp analyzer (Seahorse Bioscience, USA) at $37^{\circ} \mathrm{C}$. The NSCs $\left(2 \times 10^{6}\right.$ cells per $\left.\mathrm{mL}\right)$ were mixed with each hydrogel and incubated in the culture medium for $24 \mathrm{~h}$. Subsequently, the medium was changed to unbuffered DMEM (DMEM containing $1 \mathrm{mM}$ sodium pyruvate, $31 \mathrm{mM} \mathrm{NaCl}, 25 \mathrm{mM}$ glucose, and $2 \mathrm{mM}$ GlutaMAX, $\mathrm{pH}$ 7.4), and incubated at $37^{\circ} \mathrm{C}$ for $1 \mathrm{~h}$. The cellular oxygen consumption rate (OCR) was determined by Seahorse XF-96 software.

\section{Gene expression for cells in CS-CNF hydrogels}

To identify the behavior of NSCs embedded in hydrogels containing with CNFs, the expression levels of NSCrelated genes [nestin, glial fibrillary acidic protein (GFAP), CNPase, $\beta$-tubulin, and microtubule-associated protein 2 (MAP2)] were determined by real-time reverse transcriptase-polymerase chain reaction (RT-PCR). Total RNA from the cells embedded in the hydrogels was extracted with the Trizol reagent (Invitrogen, Carlsbad, CA, USA). cDNA was obtained from RNA by reverse transcription with the RevertAid First Strand cDNA Synthesis Kit (MBI Fermentas, St. Leon-Rot, Germany). RT-PCR was performed by the Applied Biosystems StepOne thermal cycler using the KAPA SYBR FAST qPCR 
Kit (KAPA Biosystems). The primer pairs of each gene used in this study are shown in Table S1 (Supplementary data). The expression level of each gene was normalized to that of glyceraldehyde 3-phosphate dehydrogenase (GAPDH), which is an internal control.

\section{Protein expression by immunostaining}

The protein expression of NSCs in CS-CNF hydrogels was analyzed by immunostaining. The ntibodies and concentrations were the same as those described previously for $3 \mathrm{D}$ immunostaining ${ }^{32,33}$. Immunostaining was performed according to the general protocol with some modifications. The NSCs $\left(2 \times 10^{6}\right.$ cells $)$ were embedded in the hydrogels with NSC medium without G418 and cultured for 7 days. For immunostaining, the hydrogels were washed with PBS and fixed with $4 \%$ paraformaldehyde solution for approximately $30 \mathrm{~min}$. After blocking with $5 \%$ bovine serum albumin (Chumeia), the NSCs were incubated with the respective antibodies (1:100 dilution) of nestin (GeneTex, GTX26142), GFAP (BioLegend, B209352), CNPase (BioLegend, B209442), $\beta$-tubulin (Proteintech, 10068-1-AP), and MAP2 (BioLegend, B206152) at $4{ }^{\circ} \mathrm{C}$ for $24 \mathrm{~h}$. After completion of the reaction, the samples were washed several times with Tween 20 (PBST) buffer, and stained with secondary goat antirabbit or anti-mouse IgG antibody (1:500 dilution; GeneTex) at $4{ }^{\circ} \mathrm{C}$ for $24 \mathrm{~h}$. The expression of each protein in the samples was observed with a Leica (DM IRB) fluorescence microscope. The fluorescence intensity of the image was quantified to compare the values of different samples.

\section{In vivo therapeutic function evaluation by zebrafish experiments}

Adult wild-type zebrafish were maintained and paired under standard protocols.

The therapeutic effects of NSCs on CNS-impaired adult zebrafish were evaluated by the cerebellum injury model of adult zebrafish ${ }^{34}$ with some modifications. The zebrafish were anesthetized with $200 \mathrm{ppm}$ tricaine (SigmaAldrich, USA). Afterward, a 26G (260 $\mu \mathrm{m}$ inner diameter) syringe needle (Terumo, Philippines) was vertically pierced into the skull of an adult zebrafish $1.5 \mathrm{~mm}$ deep to cause cerebellum injury. After stabbing, cells (approximately $1 \times 10^{4}$ cells total) in hydrogels $(1 \mu \mathrm{L})$ were injected into the wound by a winged infusion set (27G; TOP, Malaysia). The functional activity (swimming speed) of adult zebrafish was analyzed from 1 to 6 days ${ }^{35}$.

\section{Statistical analysis}

All the quantitative values from the experiments are expressed as the mean \pm standard deviation. In an individual experiment, each group contained multiple samples $(n=3-5)$. To confirm reproducibility, each experiment was repeated three times. Statistical differences among the experimental groups were determined by one-way analysis of variance (ANOVA). A $p$ value $<$ 0.05 was considered statistically significant.

\section{Results}

Self-healing and injectable properties of CS-CNF hydrogels

The CNFs were prepared by TEMPO-oxidation of cellulose and had an oxidation (substitution) ratio of approximately $30 \%{ }^{25}$. The average diameter of the dispersed CNFs was approximately $3 \mathrm{~nm}$, and the length was in the range of $10-20 \mu \mathrm{m}$ (Fig. S1, Supplementary data). The composite hydrogels of CS and CNFs were further prepared to contain different contents of CNFs $(0.06,0.09$, 0.12 , and $0.15 \mathrm{wt} \%$ ) (abbreviated as CS-CNF1, CS-CNF2, CS-CNF3, and CS-CNF4, respectively), as shown in Fig. 1A. The macroscopic flow and self-healing properties of various CS-CNF nanocomposite hydrogels are shown in Fig. 1B. For the pristine CS self-healing gel, the central hole $(0.5 \mathrm{~cm})$ diminished with time and finally disappeared after $12 \mathrm{~h}$. Meanwhile, the central holes in the CS-CNF nanocomposite hydrogels were fully recovered in only $9 \mathrm{~h}$. Based on visual observation, the self-repairing rate of the CS-CNF nanocomposite hydrogels was faster than that of the pristine CS hydrogel. In addition, the CS-CNF hydrogels could be injected easily through a $160 \mu \mathrm{m}$ diameter syringe needle without clogging, while the pristine CS hydrogel could only be injected by being pushed hard through the $160 \mu \mathrm{m}$ diameter syringe and fragmented easily. The surface of $160 \mu \mathrm{m}$-needle-injected hydrogels was smooth for the composite hydrogels, particularly for CS-CNF2 and CS-CNF3 hydrogels (Fig. 1B).

\section{Rheological properties of CS-CNF hydrogels}

The rheological properties of CS and CS-CNF2 hydrogels are compared in Fig. 2. When equilibrated at $37^{\circ} \mathrm{C}$ for approximately $2000 \mathrm{~s}$, the storage shear modulus $\left(G^{\prime}\right)$ for both CS and CS-CNF2 reached a steady value and was approximately $2 \mathrm{kPa}$ (Fig. 2A, B). The dynamic strain sweep experiment conducted at $1 \mathrm{~Hz}$ showed that the CS hydrogel underwent a gel-to-sol transition $\left(\mathrm{G}^{\prime}\right.$ reduced from 2 to $0.04 \mathrm{kPa}$ ) at an approximately $285 \%$ strain, while CS-CNF2 became sol at a much lower strain (52\%) (Fig. 2C, D). In addution, the dynamic strain sweep data of CS-CNF2 (Fig. 2D) revealed that the transition from gel to sol occurred quite suddenly and there was a sharp drop in $G^{\prime}$ when the strain reached approximately $50 \%$. A reversible sol-gel transition was revealed by the damage and healing cycles (Fig. 2E, F). Both hydrogels were completely recovered from damage upon the step change in oscillatory strain. The rheological properties of the other CS-CNF nanocomposite hydrogels are shown in Fig. S2 (Supplementary data). The storage modulus $\left(G^{\prime}\right)$ and the minimal strain for the gel-to-sol transition of the 


\section{A}

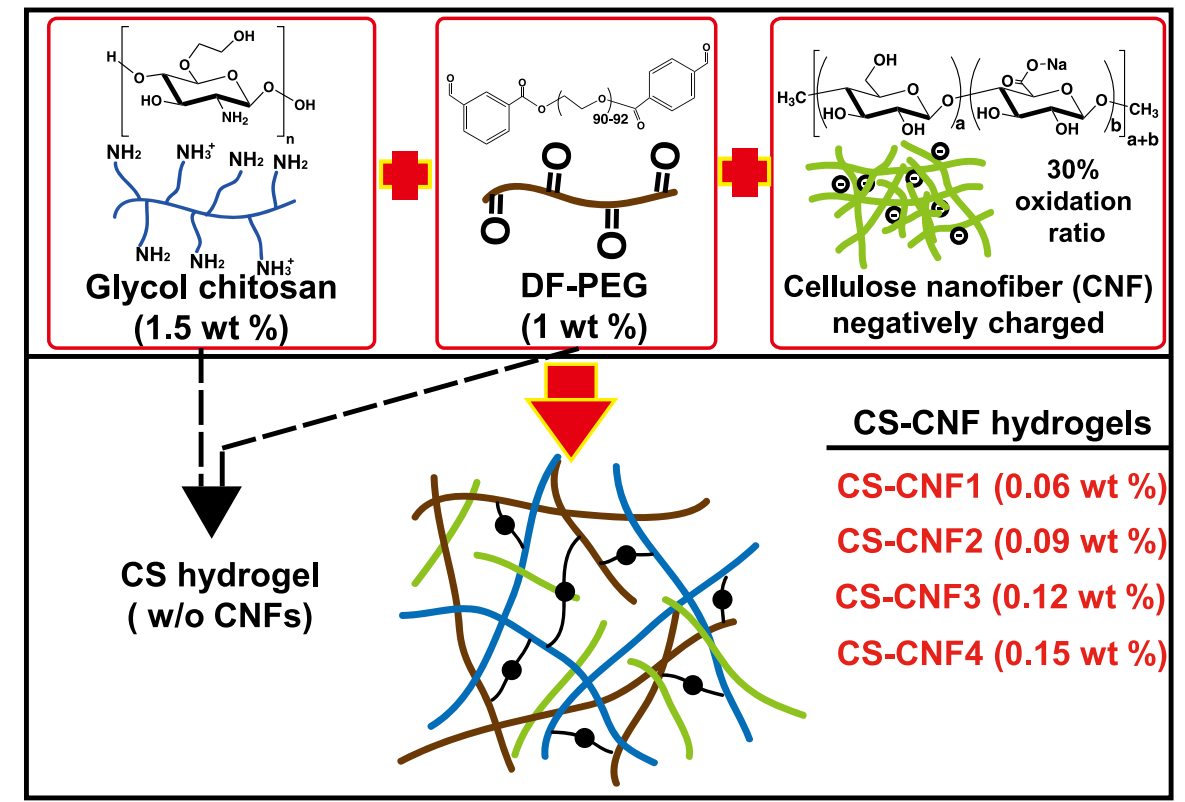

B
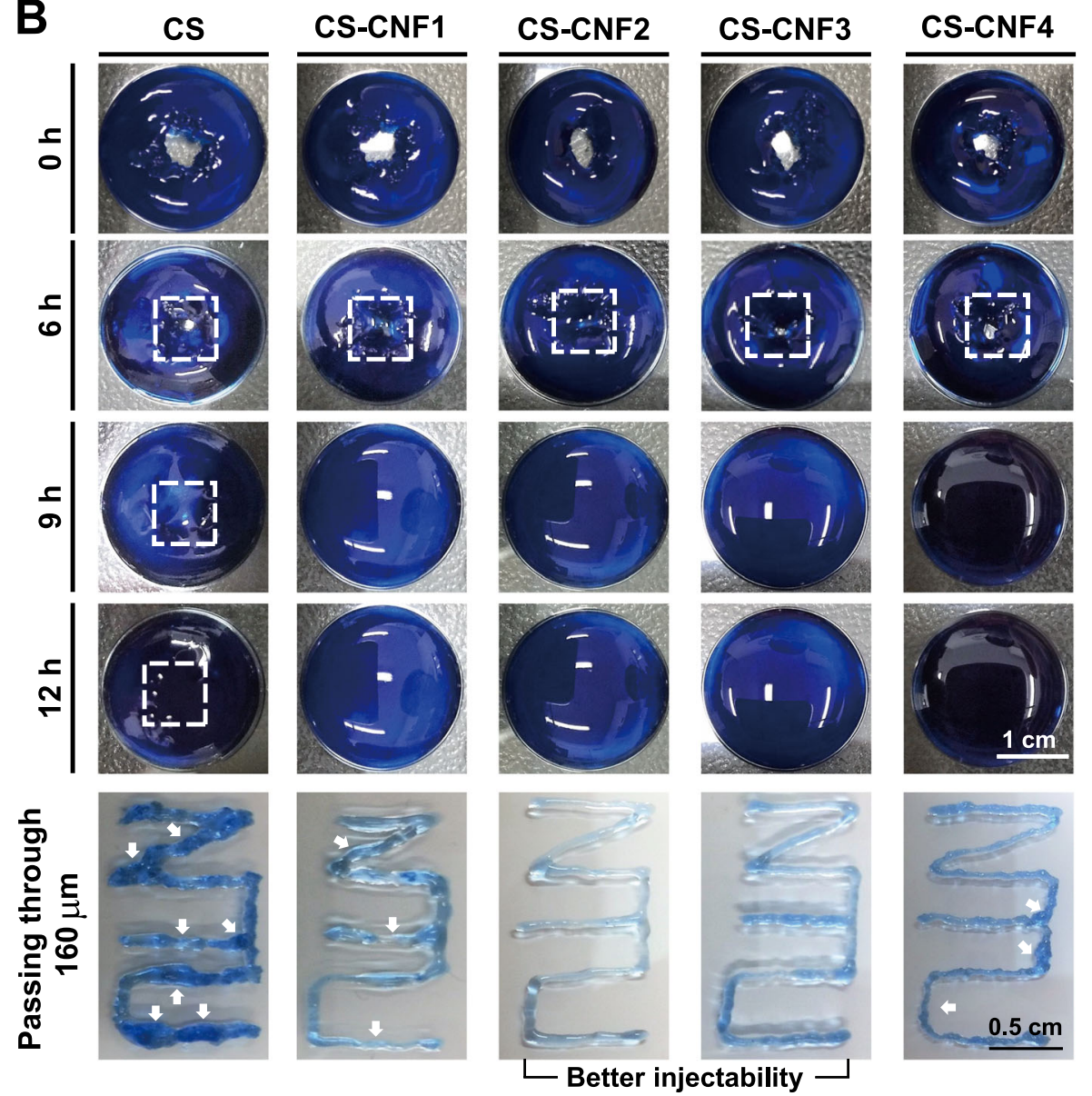

Fig. 1 (See legend on next page.) 
(see figure on previous page)

Fig. 1 Preparation of CS-CNF self-healing hydrogels. A Benzaldehydes of difunctionalized PEG (DF-PEG) were mixed with cellulose nanofibers (CNFs), followed by the addition of glycol CS to form a self-healing hydrogel. B Gross observation of self-healing by disappearance of the central holes in the hydrogels, and by injection through a $160 \mu \mathrm{m}$ diameter needle (30G). The hydrogels were stained with trypan blue to aid visual examination. CS-CNF2 and CS-CNF3 could pass through the needle without clogging, but CS, CS-CNF1, and CS-CNF4 hydrogels could not pass continuously and were fragmented. The broken line boxes indicate that the central hole had not completely sealed. The white arrows indicate gel incontinuity or gel fragmentation

hydrogel are summarized in Table 1. For CS-CNF hydrogels with a low (CS-CNF1) or high (CS-CNF3 and CS-CNF4) CNF content, the critical strain required for destroying the hydrogel was greater than that for CS-CNF2. At the highest content of CNF (0.15 wt\%), the hydrogel (CS-CNF4) did not fully recover its original strength (i.e., recovered only approximately $60 \%$ ).

\section{Thermal analyses and degradation studies of CS hydrogels and CS-CNF nanocomposites}

The pristine CS hydrogel and CS-CNF2 hydrogel were decomposed at approximately 250,350 and $400{ }^{\circ} \mathrm{C}$, according to TGA curves of the self-healing hydrogels shown in Fig. S3A. The thermal stability of the CS-CNF2 hydrogel was better than that of the pristine CS hydrogel. The remaining fractions of the CS hydrogel at 250, 350 and $400{ }^{\circ} \mathrm{C}$ were approximately $83 \%, 70 \%$, and $40 \%$, while those of the CS-CNF hydrogel were $83 \%, 70$, and $45 \%$, respectively. The degradation rates for hydrogels in PBS at $37^{\circ} \mathrm{C}$ are displayed in Fig. S3B. All hydrogels degraded significantly in 28 days. In 3 days, the CS and CS-CNF4 hydrogels revealed approximately $20 \%$ weight loss. Meanwhile, CS-CNF1, CS-CNF2 and CS-CNF3 showed weight loss less than $20 \%$ even after 14 days. The degradation of CS-CNF2 was the slowest, with approximately $40 \%$ degraded at 21 day and approximately $85 \%$ degraded at 28 day.

\section{Evaluation of NSC viability in nanocomposite hydrogels by confocal microscopy and CCK-8 assay}

The long-term proliferation of NSCs in different hydrogels was evaluated by the Cell Counting Kit-8 (CCK8 ) assay, and the results are demonstrated in Fig. 3A. At 3 day, the numbers of NSCs were not significantly different in the pristine CS hydrogel and CS-CNF nanocomposite hydrogels. After 7 days, the number of NSCs in the pristine CS hydrogel was lower than that in any of the CS-CNF groups. At 21 day, CS-CNF2 had the greatest number of NSCs. At 28 day, the number of NSCs somewhat declined in all groups.

Cell viability in hydrogels was confirmed at 3 day by confocal microscopy (Fig. 4). Cells in CS-CNF2 were more intact (approximately 82.3\%) than those in CS and CS-CNF4 (approximately 72\%). Meanwhile, CNFs in hydrogels were self-assembled, and the nanofeatures of the fibers were maintained.

Aerobic metabolism of NSCs in nanocomposite hydrogels

The bioenergetics of NSCs in hydrogels was evaluated by aerobic metabolism and is represented by the OCR values of the cells, as shown in Fig. 3B. The quantitative indices including basal OCR values, OCR associated with ATP production, and OCR associated with nonmitochondrial oxygen consumption, were defined from the bioenergetic profiles, as illustrated in Fig. S4. The basal OCR value (before the addition of oligomycin) for cells in CS-CNF2 was higher than that for cells in the pristine CS hydrogel. The oxygen consumption for cells in CS-CNF2 increased continuously after the addition of carbonyl cyanide p-(tri-fluromethoxy)phenyl-hydrazone (FCCP, mitochondrial uncoupler), indicating the elevated efficiency of mitochondrial electron transfer. In contrast, a continuous increase was not observed in the CS group. After the addition of rotenone, the nonmitochondrial oxygen consumption of the CS-CNF2 group was also higher than that of the CS group. Additional results showing the metabolism in other self-healing hydrogels are displayed in Fig. 3C-E. Evidently, oxygen metabolism was highly associated with the self-healing properties of the hydrogels. Both mitochondrial function and nonmitochondrial respiration were significantly increased in CS-CNF2 and decreased in CS-CNF4, compared to those in the pristine CS hydrogel.

\section{Evaluation of neural differentiation of NSCs in nanocomposite hydrogels by gene expression}

The effects of the nanocomposite hydrogels on NSC differentiation are shown in Fig. 5. After 3 days, NSCs embedded in CS-CNF2 expressed a greater level of the stemness gene (nestin) than those cultured on the conventional $2 \mathrm{D}$ tissue culture polystyrene dish (TCPS) as well as those in the pristine CS hydrogel and the other CS-CNF hydrogels. At 14 day, the gene expression of nestin was the greatest for NSCs in CS-CNF1, CS-CNF2, and $\mathrm{CS}-\mathrm{CNF} 3$, while there was no significant difference among the three. The gene expression level of GFAP was significantly upregulated only after 14 days and was the highest for NSCs in CS-CNF2 and CS-CNF3. The gene 

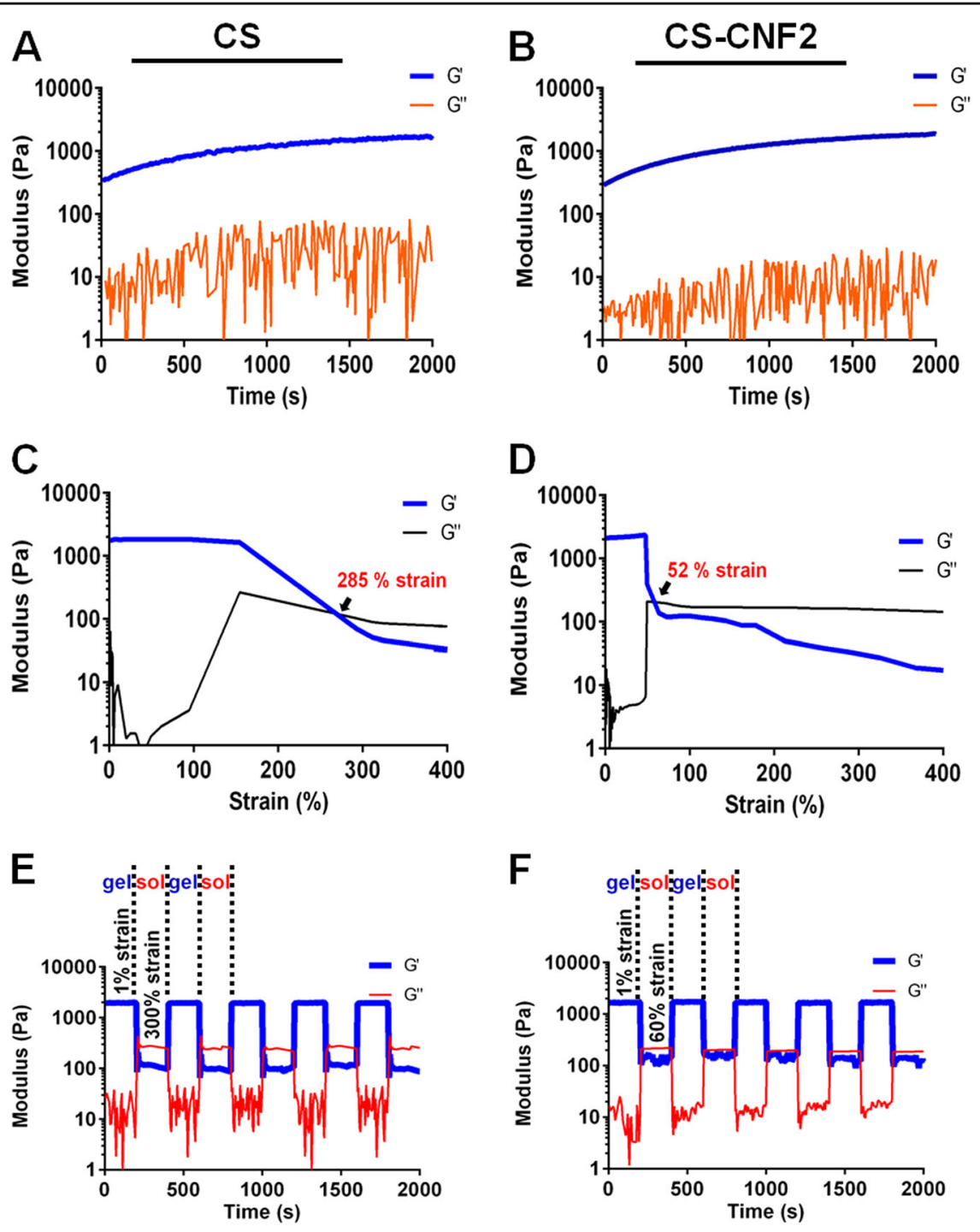

Fig. 2 Rheological properties of the pristine CS hydrogel and CS-CNF2 hydrogel. A, B The moduli $\left(G^{\prime}\right.$ and $\left.G^{\prime \prime}\right)$ of the hydrogel at $37^{\circ} \mathrm{C}(1 \%$ and $1 \mathrm{~Hz})$ against time. C, D Strain sweep experiments for the equilibrated hydrogels at $37^{\circ} \mathrm{C}(1 \mathrm{~Hz}) . \mathbf{E}, \mathbf{F}$ The damage-healing cycles of the hydrogels conducted by introducing continuous step changes in strain ( $1 \%$ strain $\rightarrow n \%$ strain $\rightarrow 1 \%$ strain $\rightarrow \ldots$ ) ( $n \geq$ the minimal strain for damage to occur) at $37^{\circ} \mathrm{C}$

expression level of CNPase was the highest in the CS-CNF1 group at 3 day and in the CS-CNF2 group at 7 and 14 day. The gene expression level of $\beta$-tubulin (an early neuronal marker) was significantly high only in the CS-CNF2 group at 3 day. From 7 to 14 days, only the CS-CNF4 group showed upregulation of $\beta$-tubulin. In contrast, the gene expression of MAP2 (a mature neuronal marker) significantly increased in the CS-CNF2 group during the culture period, followed by that in the CS-CNF1 and CS-CNF3 groups. The effects of CNFs (alone) on NSC differentiation are shown in Supplementary Fig. S5A. After 3 days, NSCs cultured on the conventional 2D TCPS expressed a greater level of $\beta$-tubulin (the early neuronal marker) than those cultured with
CNFs. Data at 3 day also suggested that NSCs cultured with CNFs may have a tendency to express more MAP2 (the mature neuronal marker) but the tendency was not as obvious. At 7 day, the gene-expression levels of Nestin, CNPase, $\beta$-tubulin, and MAP2 were significantly upregulated for NSCs cultured with CNFs.

\section{Protein expression of NSCs in nanocomposite hydrogels}

The protein expression levels of neural-related biomarkers were evaluated by immunostaining and are shown in Fig. 6A. After 7 days, the protein expression levels of nestin, GFAP, CNPase, and MAP2 in CS-CNF2 appeared to be greater than those in the pristine CS, especially for MAP2. The expression levels quantified by 

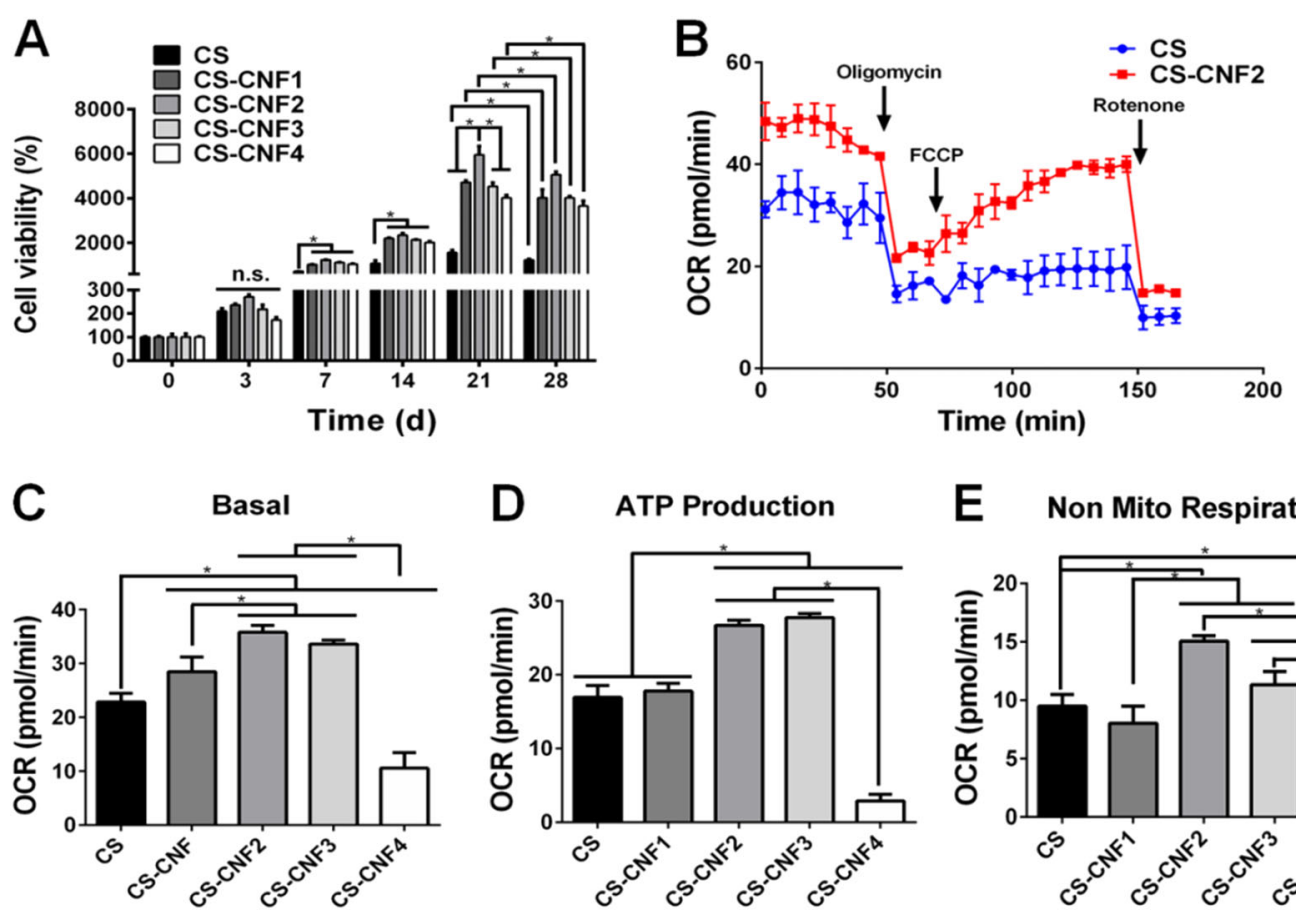

E Non Mito Respiration

Fig. 3 Cell growth and oxygen metabolism of NSCs in different hydrogels. A Long-term cell proliferation in the hydrogels was measured by the CCK-8 assay and is expressed as cell viability. The cell viability was normalized to the value at 0 day (in each hydrogel) and is represented as the percentage (\%). B The oxygen consumption rate (OCR) as a parameter to evaluate the oxygen metabolism of the cells inside hydrogels. The inhibitors oligomycin, FCCP, and rotenone, were added as indicated. C-E Comparison of the OCR indices for NSCs in different self-healing hydrogels: C basal OCR, D OCR associated with ATP production and $\mathbf{E}$ nonmitochondrial OCR. The basal OCR (C) and ATP production (D) serve as indices of mitochondrial function. n.s. not significant. ${ }^{*} p<0.05$ among the indicated groups

Table 1 The storage modulus $\left(G^{\prime}\right)$ and the minimal strain for gel-to-sol transition to occur, based on rheological measurements at $1 \mathrm{~Hz}$

\begin{tabular}{lll}
\hline Hydrogel & Storage modulus (Pa) & $\begin{array}{l}\text { Strain of gel-to-sol } \\
\text { transition (\%) }\end{array}$ \\
\hline $37^{\circ} \mathrm{C}$ & & \\
$\mathrm{CS}$ & 1823 & 285 \\
$\mathrm{CS}-\mathrm{CNF} 1$ & 1766 & 156 \\
$\mathrm{CS}-\mathrm{CNF2}$ & 1713 & 52 \\
$\mathrm{CS}-\mathrm{CNF3}$ & 1550 & 62 \\
$\mathrm{CS}-\mathrm{CNF} 4$ & 1522 & 70 \\
$25^{\circ} \mathrm{C}$ & & \\
$\mathrm{CS}-\mathrm{CNF2}$ & 1816 & 56 \\
\hline
\end{tabular}

the fluorescence intensities (\%) of the staining are shown in Fig. 6B. Based on the data, the protein-expression levels of nestin, GFAP, CNPase, and MAP2 for NSCs in the pristine CS hydrogel were lower than those in the CS-CNF2 hydrogels. In addition, the protein-expression levels of GFAP and MAP2 for NSCs in the pristine CS hydrogel were lower than those in the CS-CNF3 hydrogel. The protein expression level of nestin showed no significant difference between the CS-CNF1 and CS-CNF2 groups, but was higher than that in- the pristine CS and other groups. No significant difference in $\beta$-tubulin expression was observed among the CS, CS-CNF1, and CS-CNF2 groups, while the $\beta$-tubulin expression level in the CS-CNF3 and CS-CNF4 groups was lower than that in the pristine CS and other groups. In particular, the cells in CS-CNF2 had the greatest CNPase and MAP2 expression among all groups. In general, the tendency of protein expression followed that of gene expression observed previously.

Implantation in the brain injury model of adult zebrafish

The therapeutic potential of NSCs embedded in the CS-CNF2 hydrogel in the cerebellar injury of adult zebrafish was first analyzed, and the results are displayed in Fig. 7A, B. The survival rates of the injured zebrafish transplanted with cell-embedded CS-CNF2 remained approximately $70 \%$ after 6 days. In contrast, only 25\% injured zebrafish in the control group survived after 2 days and less than 20\% survived after 6 days (Fig. 7A). With regard to locomotion, the cerebellum-injured 


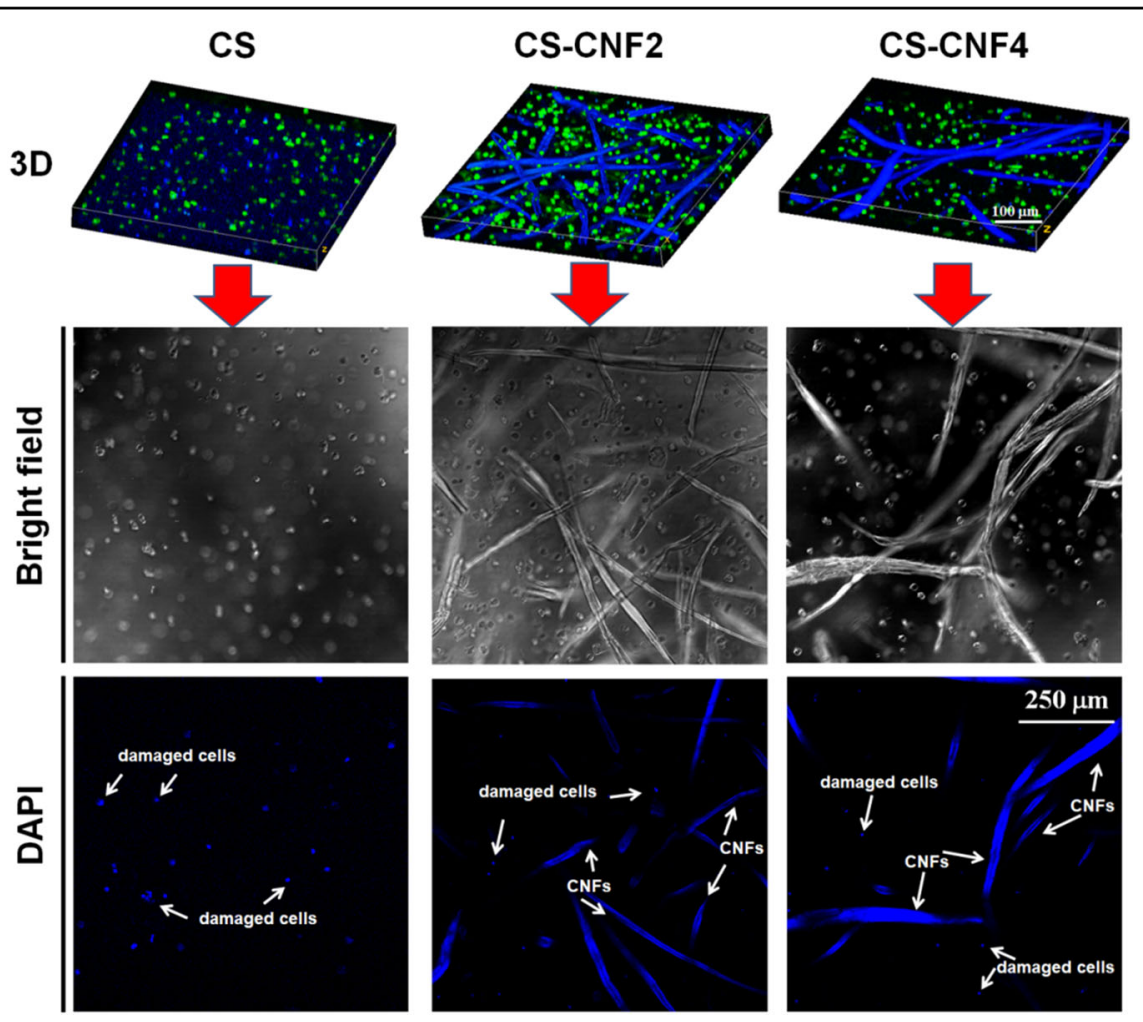

Fig. 4 The viability of NSCs in hydrogels visualized by confocal microscopy. At 3 day, there were more damaged cells (blue spots) in the pristine CS hydrogel (approximately 72.6\% viability) and CS-CNF4 hydrogel (approximately 71.5\% viability) than in the CS-CNF2 hydrogel (approximately 82.3\% viability). The CNFs were self-assembled in the hydrogels, forming a network. Meanwhile, the nanofeatures of CNFs were maintained. The top 3D images were acquired from $100 \mu \mathrm{m}$ thick hydrogels $(850 \mu \mathrm{m} \times 850 \mu \mathrm{m}$ area) for photography. The 2D images below represent data from a slice that was only $10 \mu \mathrm{m}$ thick (a single slice in the Z-stack)

zebrafish transplanted with the cell-embedded CS-CNF2 hydrogel recovered $45 \%$ and $65 \%$ of swimming activity after 4 and 6 days, respectively. While those without any transplantation demonstrated only $20 \%$ recovery in swimming activity during the same period (Fig. 7B). These data suggested the better regeneration effect of NSCs embedded in the composite self-healing hydrogel. The therapeutic potential of NSCs with CNFs in cerebellar injury of adult zebrafish is displayed in the Supplementary Fig. S5B. For the injured zebrafish transplanted with CNFs containing NSCs, only 33\% survived after 2 days and less than $20 \%$ survived after 4 days. With regard to locomotion, only $25 \%$ and $35 \%$ of the swimming activity was recovered after 2 and $4 \mathrm{~d}$, respectively, in the cerebelluminjured zebrafish transplanted with CNFs containing NSCs. Based on these results, the effect of CNFs alone was not significant in vivo.

\section{The effect of self-healing ability of hydrogels on the neuroregeneration of zebrafish}

We performed additional experiments to compare the therapeutic effect of NSC-embedded self-healing hydrogels. The effect of self-healing on the therapeutic potential of the cell-embedded hydrogels in the zebrafish brain injury model is summarized in Fig. 7C. After 6 days, the recovery of the swimming activity was ranked in the order of CS-CNF2 (65\%) CS-CNF3 $(60 \%)>$ CS-CNF1 $(42 \%)$ $\sim \mathrm{CS}-\mathrm{CNF} 4(38 \%) \sim \mathrm{CS}$ (36\%). The tendency of the hydrogel neuroregeneration potential was generally in accordance with the degree of strain sensitivity (i.e., readiness for reversible structure damage-healing) of the hydrogels.

\section{Discussion}

The literature has revealed that the mechanical properties of self-healing hydrogels could be adjusted by the chemical composition, solid contents, or type of composite hydrogels ${ }^{15,36-38}$. Very recently, the self-healing properties of poly(2-acrylamido-2-methyl-1-propanesulfonic acid) hydrogels were also found to be tunable ${ }^{39}$. However, reports regarding adjustments to extend selfhealing are still very limited. Moreover, the biocompatibility and biodegradability of such hydrogels have not been examined. Therefore, the exact relationship between self-healing and biocompatibility remains unexplored in the area of hydrogels. In the current study, we developed a 

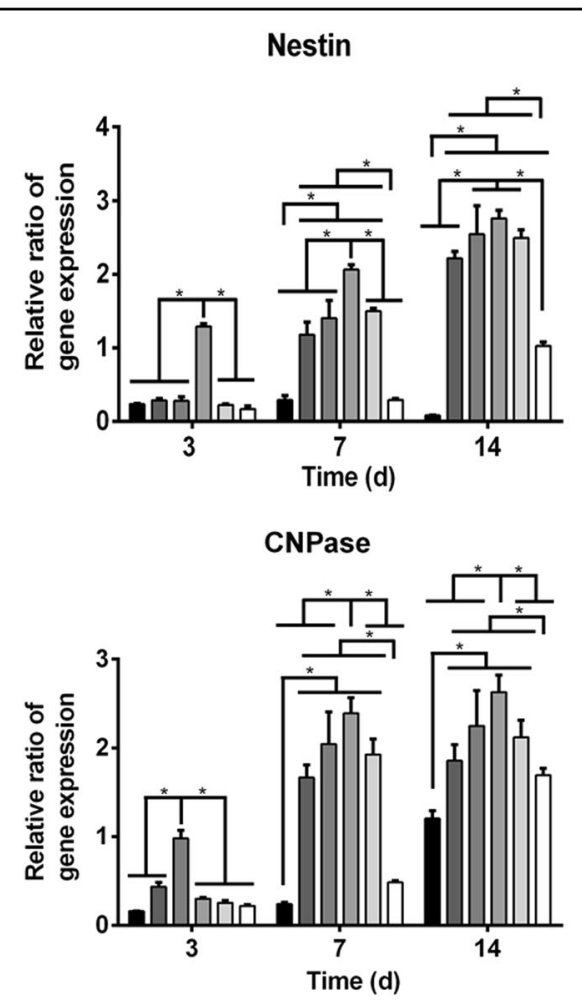

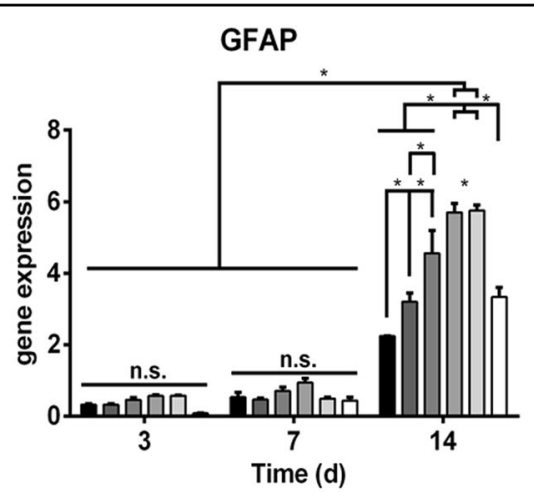

$\beta$-Tub

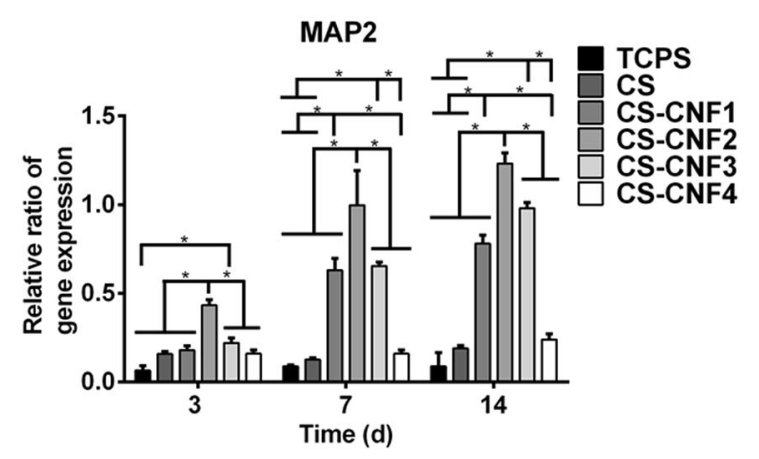

Fig. 5 Gene expression of NSCs embedded in different hydrogels for 3, 7, and 14 days. ${ }^{*} p<0.05$ among the indicated groups

series of CS self-healing hydrogels by making nanocomposite hydrogels from CS and CNFs. CNFs strongly interact and entangle among the fibrils. Meanwhile, CNFs are long, flexible, and can readily form hydrogen bonds with the surrounding ${ }^{40}$. Moreover, the addition of CNFs may cause a significant steric hindrance effect and thereby modulate the dynamic Schiff bonding of the hydrogel. We expected that the mechanical and self-healing properties may vary in the CNF composite hydrogels. These hydrogels may offer a model system for correlating the self-healing function of hydrogels with their biological performance.

The Schiff base is a double bond of carbon-nitrogen comprised by nucleophilic attack. Several self-healing hydrogels have been exploited by aromatic Schiff base ${ }^{41}$ or aliphatic Schiff base ${ }^{42}$. The aromatic Schiff base, as is the case in the current study, generally shows greater stability. The rheological properties of the composite hydrogels were first analyzed. To our surprise, the mechanical properties of strength and modulus did not increase in the nanocomposite hydrogels. In addition, the strain sensitivity and self-healing rate revealed a biphasic change. In the strain sweep experiment, the $\mathrm{G}^{\prime}$ value of the pristine CS hydrogel gradually decreased beyond approximately $285 \%$ strain. The drop in $G^{\prime}$ upon strain increase was more rapid as the amount of CNFs increased until an almost vertical drop of $\mathrm{G}^{\prime}$ was observed in CS-CNF2 (0.09 wt\% CNFs) at approximately $52 \%$ strain. Beyond this concentration $(0.09 \mathrm{wt} \% \mathrm{CNFs})$, the trend was reversed, i.e., the $\mathrm{G}^{\prime}$ drop was slower and the critical strain for selfhealing increased. Because of these nonmonotonic changes, we hypothesized that apart from the Schiff-base 


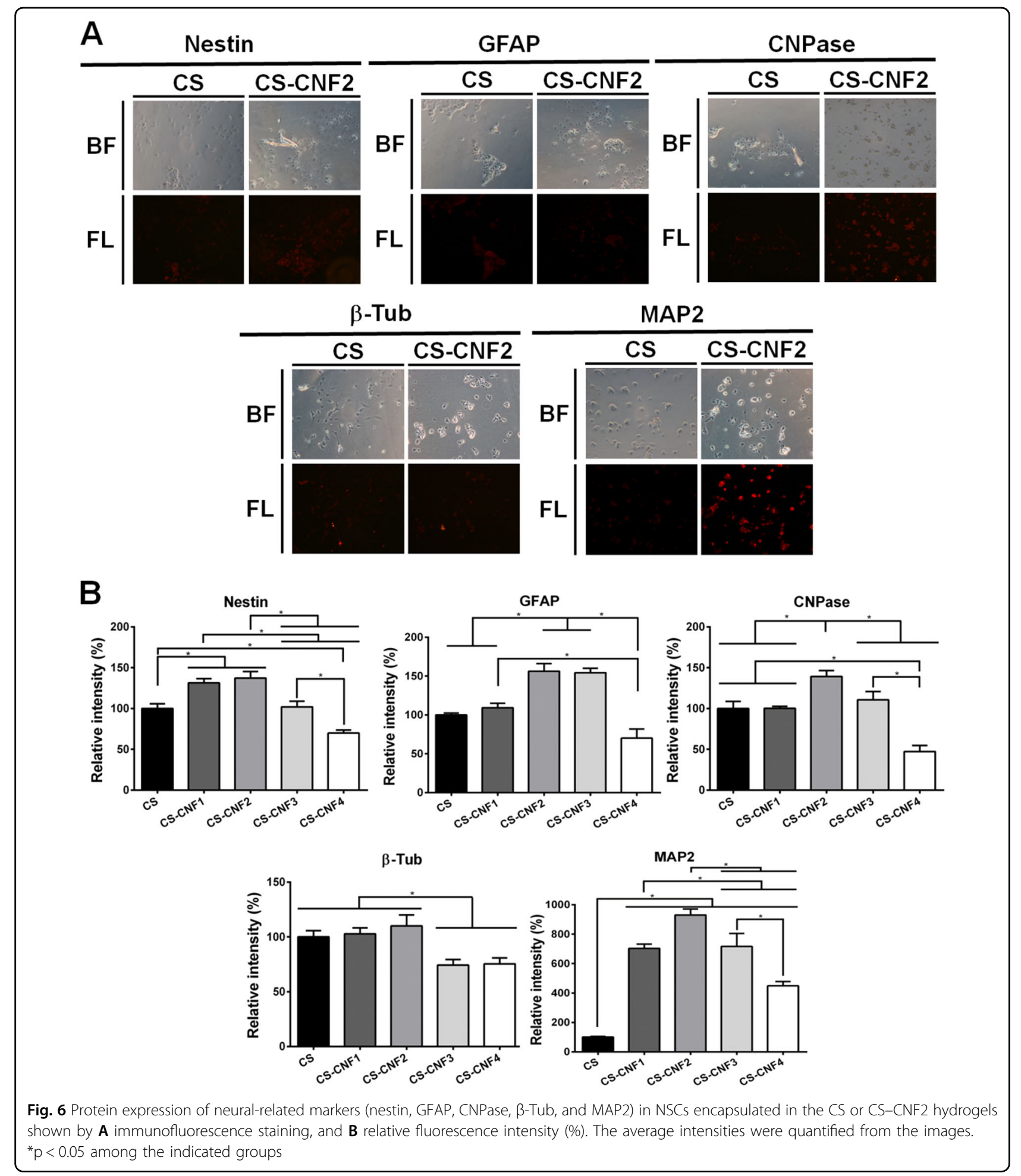

dynamic bonding of DF-PEG, there may be additional interactions in this system such as the electrostatic interaction between the positive charge on the glycol CS and the negative charge of CNFs added ${ }^{43,44}$. The ionic network as well as the fibrous structure of $\mathrm{CNFs}^{45,46}$ may have caused steric hindrance effects while Schiff-base bonds formed and undeformed, leading to changes in selfhealing. In long-term measurements using a small strain, the modulus and strength of the composite hydrogels did not change much probably because the Schiff-base was 

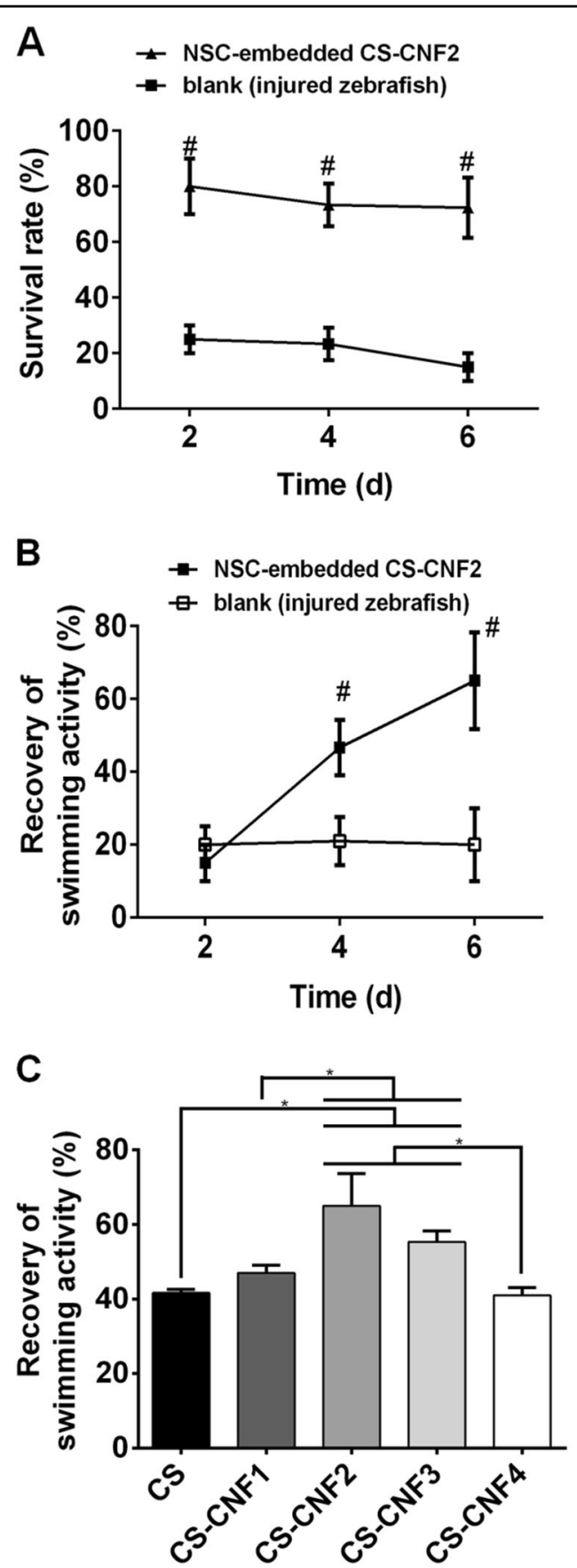

Fig. 7 The functional recovery of adult zebrafish after CNS injury and injection of CS-CNF hydrogels embedding NSCs. A Survival rate and $\mathbf{B}$ swimming activity of zebrafish receiving NSC-embedded CS-CNF2 hydrogel during a period of 6 days. The swimming activity of wild type zebrafish was considered as $100 \%$. C Comparison of the swimming function recovery among different hydrogels (containing NSCs). \# $<0.05$ relative to the blank group (A, B). ${ }^{*} p<0.05$ among the indicated groups $(\mathbf{C})$ more dominant than the electrostatic force and the steric effect. While the pristine CS hydrogel was destroyed by a large strain, the Schiff-base bonds broke slowly and transformed into sol. When CNFs were present in the hydrogel before reaching the saturation amount, the additional electrostatic forces and steric hindrance would interfere with the Schiff-base dynamics in the hydrogel, at a critical strain (approximately 50\% strain), the Schiff-base bonds suddenly collapsed to transform the hydrogel into sol. In the case of a high percentage of CNFs (approximately $0.15 \mathrm{wt} \% \mathrm{CNFs}$ ), the previous structure in the destroyed nanocomposite hydrogel may not be fully recovered within a specific time period because of overwhelming electrostatic interactions and steric effects. By the above mechanism, the self-healing property of the CS hydrogel may be tuned by the concentration of CNFs, but not proportionally. Moreover, the addition of CNFs did not change the dynamic modulus of the hydrogel at low strains, possibly because of the multiple interaction forces between CS and CNFs. Furthermore, injectable hydrogels with self-healing properties could have a longer lifespan, which is beneficial for tissue regeneration ${ }^{47}$.

One of the advantages of the current hydrogel model is that the self-healing properties of the CS-CNF hydrogels were independent of their rigidity (modulus) of the hydrogels. In this study, all CS and CS-CNF hydrogels had similar modulus $\left(\mathrm{G}^{\prime}\right)$ values. In the literature, the addition of NCC (BNC or NCC, 1.5-3.5 wt\%) into alginate could increase the modulus of the hydrogel ${ }^{26,48-50}$. Our nanocomposite hydrogels did not show such an increase in modulus, probably because of the relatively small amount of CNFs $(\leq 0.15 \mathrm{wt} \%)$ added, or the competing mechanism of the Schiff-base, electrostatic, and steric interactions in our system as described in the previous paragraph. Since gel rigidity could also affect cell behavior and biological performance, keeping $G^{\prime}$ values the same for all hydrogels guaranteed that any difference in biocompatibility was not attributed to the difference in gel rigidity.

The content of CNFs to achieve optimized interactions for self-healing was $0.09 \mathrm{wt} \%$ (or between 0.09 and $0.12 \mathrm{wt}$ $\%$ ) in the CS hydrogel. Based on our calculation (Supplementary Table S2), the concentration of $\mathrm{COO}^{-}$in the suspension of CNFs $(0.09 \mathrm{wt} \%)$ was $1.8 \times 10^{-3} \mathrm{mN}$. The pKa of glycol CS was $6.5^{51}$, the concentration of $-\mathrm{NH}_{2}$ was $5.7 \times 10^{-2} \mathrm{mN}$, and the concentration of $-\mathrm{NH}_{3}^{+}$ (after protonation) on the glycol CS was $1.8 \times 10^{-4} \mathrm{mN}$ before being made into hydrogel. The concentration of aldehyde in DF-PEG was $5.0 \times 10^{-3} \mathrm{mN}$. Therefore, in the CS hydrogel, the amount of $\mathrm{NH}_{2}$ available for crosslinking was quite sufficient (ten times higher than that of aldehyde). When CNFs were added to the CS hydrogel, the protonation of $\mathrm{NH}_{2}$ may be increased, driven by the interaction between $\mathrm{COO}^{-}$and $\mathrm{NH}_{2}$. This protonation 


\section{Glycol chitosan DF-PEG}
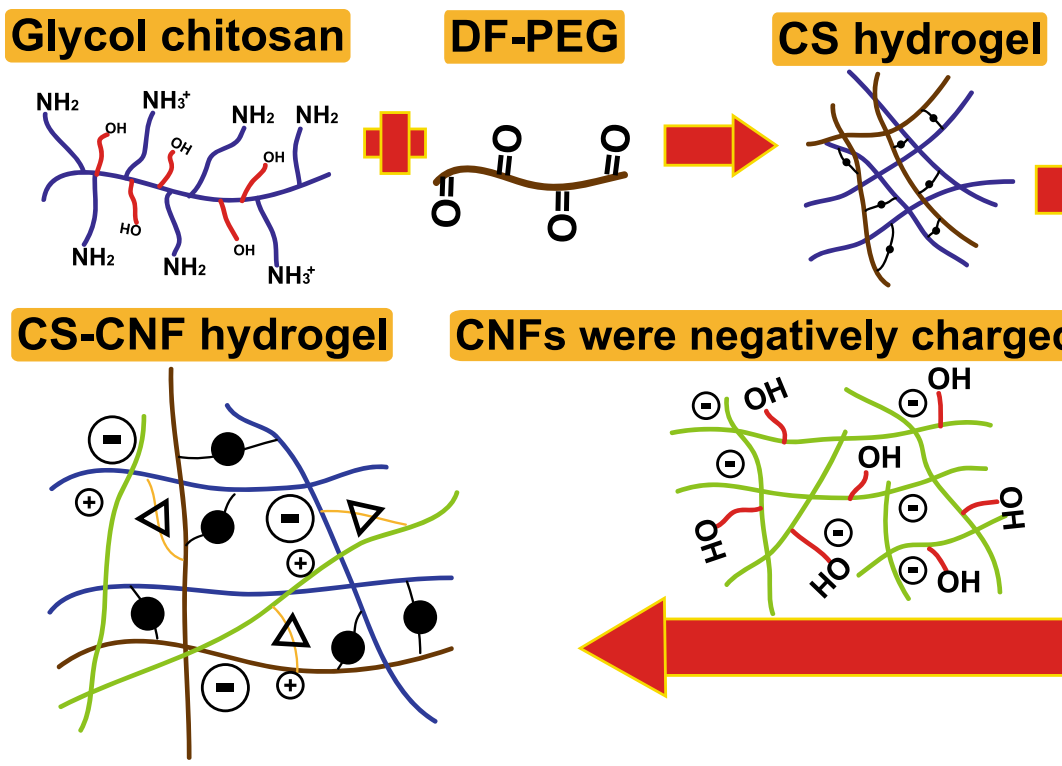

CNFs were negatively charged

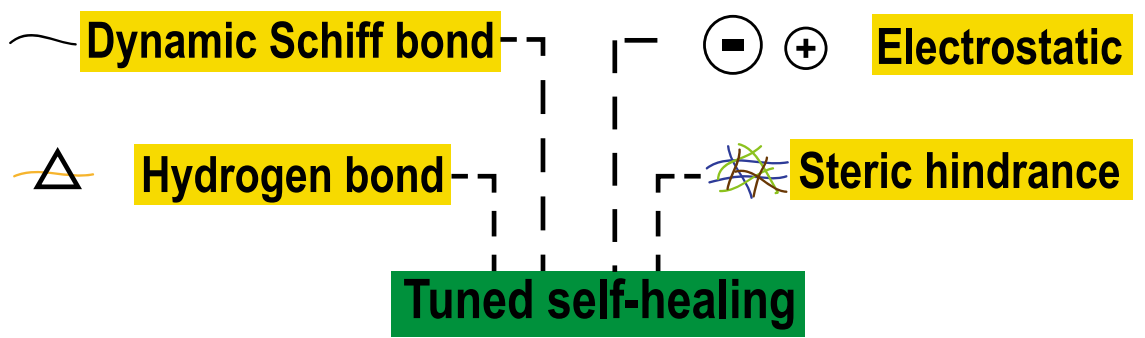

Fig. 8 The hypothetical mechanism of the self-healing properties of CS-CNF hydrogels. The addition of CNFs increased the electrostatic effect, steric hindrance, and hydrogen bonding that either compete or compensate for the dynamic Schiff-base reaction of the hydrogel to tune the selfhealing properties

would sacrifice the availability of $\mathrm{NH}_{2}$ for dynamic Schiff bonding only to a small extent. However, the steric hindrance of CNFs may also prevent the Schiff reaction. Additionally, the coulombic force was enhanced by a significant level. The electrostatic interaction may compensate for the gelling network. If the CNFs concentration was $0.15 \mathrm{wt} \%$, the overloaded CNFs with abundant hydrogen bonds, electrostatic interactions, and steric hindrance may significantly interfere with the reversible self-healing property of the hydrogel. The hypothetical mechanism for tuning the self-healing of CS by CNFs is depicted in Fig. 8. The mechanism was partially supported by infrared (IR) spectroscopy (Fig. S6, Supplementary data). We compared the spectra of the pristine CS hydrogel formed from $1.5 \mathrm{wt} \%$ glycol CS and $1 \mathrm{wt} \%$ difunctionalized PEG and those containing two different contents of CNFs $(0.09 \mathrm{wt} \%$ for CS-CNF2 and $0.15 \mathrm{wt} \%$ for CS-CNF4). In each hydrogel, a broad and intense IR absorption band in the region between 3000 and $3600 \mathrm{~cm}^{-1}$ was assigned to $\mathrm{O}-\mathrm{H}$ stretching. The peaks at 1660 and $1110 \mathrm{~cm}^{-1}$ were attributed to the stretching of
$\mathrm{C}=\mathrm{N}$ and $\mathrm{C}-\mathrm{O}$, respectively. The pristine CS hydrogel expressed greater peak absorption than did CS-CNF2 and CS-CNF4 at 3460, 1653, and $1095 \mathrm{~cm}^{-1}$. We, therefore, hypothesized that when the amount of CNFs increased, the overloaded CNFs with abundant hydrogen bonds, electrostatic interactions, and steric hindrance may significantly interfere with the reversible self-healing property of the hydrogel.

Previous literature suggest that cells embedded in selfhealing hydrogels have the ability to differentiate and proliferate and may be applied for angiogenesis ${ }^{18}$, neural regeneration $^{16,52}$, and cell delivery ${ }^{53}$. These earlier studies examined the biological functions of self-healing hydrogels with different chemical compositions and rigidity. In the current study, the long-term viability of NSCs in composite hydrogels with the same rigidity and different self-healing properties were investigated systematically. The proliferation of NSCs was found to be the greatest in CS-CNF2 that had the best self-healing property. We assumed that cell proliferation was possibly correlated with the nutrient and oxygen supply in the hydrogel. 
When cells divide during growth, the cytoskeleton has to produce strain $^{54,55}$, which may destroy the Schiff bond and increase the mass transport nearby. Therefore, hydrogels with better self-healing properties may possibly promote diffusion and mass transfer in the vicinity while providing more nutrients and oxygen for cell growth. This phenomenon may account for the positive correlation between the self-healing property and cell proliferation. The cell viability at 3 day was confirmed by confocal microscopy (Fig. 4). Cells in CS-CNF2 were more intact than those in CS and CS-CNF4. At 28 day, the number of NSCs in all hydrogels decreased. We speculated that the hydrogels were mostly degraded at this time and that a large number of cells may have fallen out of the hydrogels. The data were consistent with regard to the time frame of degradation for these hydrogels.

Although the addition of CNFs may sacrifice the dynamic Schiff bonding formed in the CS hydrogel and introduce steric hindrance, the electrostatic interaction provided a greater secondary force to establish the hydrogel networks. Therefore in the initial state, the degradation rate of the CS-CNF hydrogels was slower than that of the CS hydrogel. As was also evident from confocal microscopy, the CNFs tended to self-assemble in hydrogels, forming a network (Fig. 4). At 14 day, the remaining weight of CS-CNF4 was similar to that of the pristine CS hydrogel, possibly because the excess interaction forces, such as electrostatic interaction, may cause $\mathrm{CNF}$ aggregation and introduce more steric hindrance and thus interfere with the structural stability of the hydrogel. In addition, CS-CNF1, CS-CNF2, and CS-CNF3 displayed relatively slower degradation rates. The better stability of these composite hydrogels may be ascribed to a balance among the multiple interaction forces.

The oxygen metabolism and mitochondrial function of NSCs embedded in CS-CNF2 were further compared with those in the pristine CS hydrogel. The literature has revealed that increased oxygen metabolism in NSCs is important for the proliferation and neural differentiation $^{56,57}$. In addition, self-healing hydrogels with porous structures and suitable swelling ratios may allow for oxygen permeability and exudate absorption, and maintain a moist environment favorable for wound healing ${ }^{58}$. The basal respiration of NSCs in CS-CNF2 was greater than that in the pristine CS hydrogel. Moreover, the relatively higher OCR values suggested that the addition of CNFs may not only increase the cellular oxygen metabolism but also promote the proliferation and differentiation of NSCs. As mentioned, the better selfhealing property of CS-CNF2 may promote the nutrient and oxygen supply, which may in turn further influence cell metabolism and differentiation. The poor self-healing properties of the pristine CS hydrogel may also explain why cells in the hydrogel had a lower level of oxygen metabolism.

Analyses of neural marker genes revealed that NSCs embedded in CS-CNF2 had a greater tendency to differentiate toward glial and neuronal cells, agreeing with the fact that an increase in oxygen metabolism was associated with differentiation ${ }^{59,60}$. The expression of GFAP, CNPase, and MAP2 (red fluorescence) was more obvious for NSCs embedded in CS-CNF2 vs. those in the pristine CS hydrogel. Based on these results, the presence of an appropriate amount of CNFs in the self-healing hydrogel could effectively promote the differentiation of NSCs within only 7 days without the use of induction medium, which was a remarkably short time. The extent of neural differentiation was again positively correlated with the self-healing properties of the hydrogels. We hypothesized that self-healing may promote the mass transport to increase the proliferation and oxygen metabolism of NSCs in the hydrogel, and enhance the differentiation of NSCs in the same manner.

The gelation time of hydrogels is important for the biomedical applications of hydrogels ${ }^{61}$. The injectability and gelation rate of cell-encapsulating materials and the survival rate of transplanted cells are common concerns in cell therapy ${ }^{59,60}$. According to our in vitro findings, the self-healing property of a hydrogel is critical to cell growth and differentiation. Using the brain injury model of adult zebrafish, we demonstrated that the damaged mobile function of injured zebrafish was better rescued by NSCs embedded in the CS-CNF2 hydrogel that had better selfhealing properties. We speculated that the CS-CNF2 hydrogel injected in the injured zebrafish may heal more quickly to occupy a proper space for tissue remodeling. Hydrogel injection was reported to facilitate the remodeling of myocardial infarcted areas and subsequent tissue repair ${ }^{62,63}$. Brain injury also requires space for tissue remodeling ${ }^{64}$. The excellent space remodeling effect as well as the facilitated nutrient transport may contribute to the positive correlation between the self-healing property and neural regeneration by the hydrogels.

In summary, the CS-CNF2 self-healing hydrogel (approximately $2 \mathrm{kPa}$ ) provided NSCs with an appropriate environment for differentiation and proliferation. Similar to the nanomaterials in most studies that tend to reinforce the mechanical strength of the hydrogel ${ }^{38,65,66}$, the CS-CNF hydrogels in our study had comparable mechanical modulus but also different self-healing properties. The hydrogels with similar stiffness and tunable self-healing properties could be employed to correlate material self-healing with the neural regeneration effect. As proposed earlier, the strain generated by cell division may make self-healing hydrogels more sol-like, facilitating diffusivity and cell metabolism. We thus suggest that hydrogels with better self-healing properties could more 


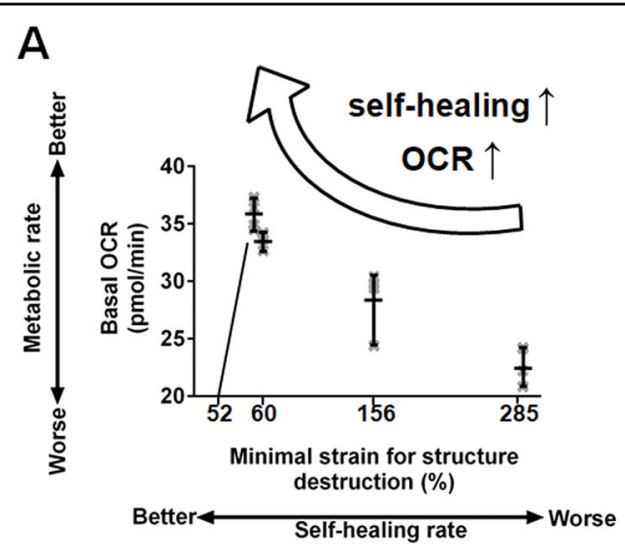

B

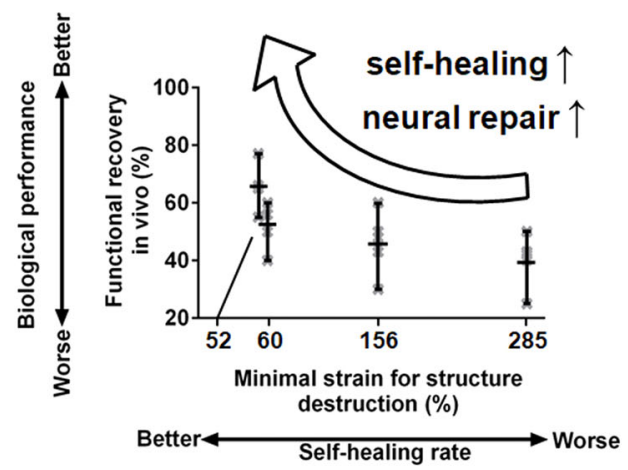

C

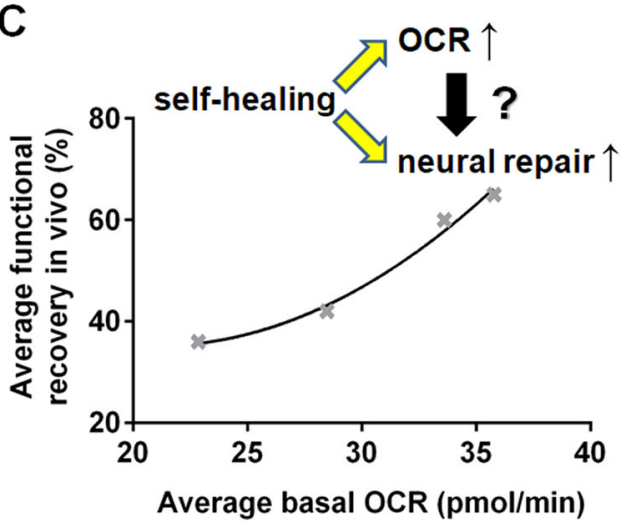

Fig. 9 The relationship between the self-healing properties of hydrogels and the functional recovery. A Relation between the metabolic rate of cells in hydrogels and the self-healing properties of hydrogels observed in this study. B Relation between the in vivo neural regeneration and the self-healing properties of hydrogels observed in this study. The symbol " $x$ " indicates the data points of the extent (\%) of functional recovery plotted against the minimal strain (\%) for structure destruction (an index of self-healing) of hydrogel. C Relation between the in vivo neural regeneration and the metabolic rate of cells in hydrogels observed in this study

effectively promote CNS functional recovery in vivo probably because of the enhanced metabolic rate, as depicted in Fig. 9. The new type of self-healing nanocomposite hydrogels with adjustable self-healing properties offer a potential direction for designing advanced biomaterials suitable for neural and various other tissue engineering applications.

\section{Conclusion}

Biodegradable CS-CNF composite hydrogels with tunable self-healing properties were established in this study. The CS-CNF composite hydrogel demonstrated the most favorable self-healing properties when it contained $0.09 \mathrm{wt}$ $\%$ CNFs. The optimized hydrogel could pass through a $160 \mu \mathrm{m}$ needle and promote oxygen metabolism and differentiation of neural stem cells. The presence of CNFs $(0.09 \mathrm{wt} \%)$ significantly increased the strain sensitivity of the self-healing hydrogel, possibly through the interaction of CNFs with the reversible Schiff bonding in the CS selfhealing hydrogel. The extra interaction also accounted for the better thermal stability and prolonged degradation of the hydrogel. The series of composite hydrogels with similar rigidity (approximately $2 \mathrm{kPa}$ ) and different selfhealing properties offered the possibility of correlating self-healing properties with the biological performance of hydrogels. The in vitro and in vivo data supported that the self-healing properties of hydrogels play a critical role in the tissue regeneration potential of the hydrogels. This study provides design rationale for self-healing hydrogels with potential biomedical applications.

\section{Acknowledgements}

This work was supported by the Program for Additive Manufacturing (MOST 106-3114-E-002-019), Ministry of Science and Technology, National Health Research Institutes (CS-107-PP-15), and National Taiwan University (NTU-CC107L891101), Taiwan, R.O.C. We thank Dr. Ing-Ming Chiu (Institute of Cellular and System Medicine, National Health Research Institutes) for providing the neural stem cells. We are also obliged to the Taiwan Zebrafish Core Facility at National Taiwan University (NTU-ERP-104R8600) for technical support.

\section{Author details}

${ }^{1}$ Institute of Polymer Science and Engineering, National Taiwan University, Taipei, Taiwan, Republic of China. ${ }^{2}$ Department of Chemical Engineering, National Chung Hsing University, Taichung, Taiwan, Republic of China. ${ }^{3}$ The Key Laboratory of Bioorganic Phosphorus Chemistry \& Chemical Biology (Ministry of Education), Department of Chemistry, Tsinghua University, Beijing, China. ${ }^{4}$ Center of Tissue Engineering and 3D Printing, National Taiwan University, Taipei, Taiwan, Republic of China. Institute of Cellular and System Medicine, National Health Research Institutes, Zhunan, Taiwan, Republic of China

Conflict of interests

The authors declare that they have no conflict of interests.

\section{Publisher's note}

Springer Nature remains neutral with regard to jurisdictional claims in published maps and institutional affiliations.

Supplementary information is available for this paper at https://doi.org/ 10.1038/s41427-019-0124-z.

Received: 14 November 2018 Revised: 14 March 2019 Accepted: 17 March 2019.

Published online: 7 June 2019 


\section{References}

1. Lee, K. Y. \& Mooney, D. J. Hydrogels for tissue engineering. Chem. Rev. 101, 1869-1879 (2001).

2. Ullah, F., Othman, M. B. H., Javed, F., Ahmad, Z. \& Akil, H. M. Classification, processing and application of hydrogels: a review. Mater. Sci. Eng. C 57, 414-433 (2015).

3. Li, L., Yan, B., Yang, J., Chen, L. \& Zeng, H. Novel mussel-inspired injectable selfhealing hydrogel with anti-biofouling property. Adv. Mater. 27, 1294-1299 (2015).

4. Zhao, X. et al. Antibacterial anti-oxidant electroactive injectable hydrogel as self-healing wound dressing with hemostasis and adhesiveness for cutaneous wound healing. Biomaterials 122, 34-47 (2017).

5. Liu, B. et al. Hydrogen bonds autonomously powered gelatin methacrylate hydrogels with super-elasticity, self-heal and underwater self-adhesion for sutureless skin and stomach surgery and E-skin. Biomaterials 171, 83-96 (2018).

6. Lu, H. D., Charati, M. B., Kim, I. L. \& Burdick, J. A. Injectable shear-thinning hydrogels engineered with a self-assembling Dock-and-Lock mechanism. Biomaterials 33, 2145-2153 (2012).

7. Cao, L. P. et al. An injectable hydrogel formed by in situ cross-linking of glycol chitosan and multi-benzaldehyde functionalized PEG analogues for cartilage tissue engineering. J. Mater. Chem. B 3, 1268-1280 (2015).

8. Zhu, D., Wang, H., Trinh, P., Heilshorn, S. C. \& Yang, F. Elastin-like proteinhyaluronic acid (ELP-HA) hydrogels with decoupled mechanical and biochemical cues for cartilage regeneration. Biomaterials 127, 132-140 (2017).

9. Mizrahy, S. \& Peer, D. Polysaccharides as building blocks for nanotherapeutics. Chem. Soc. Rev. 41, 2623-2640 (2012).

10. Suh, J. K. \& Matthew, H. W. Application of chitosan-based polysaccharide biomaterials in cartilage tissue engineering: a review. Biomaterials 21, 2589-2598 (2000).

11. Ishihara, M. et al. Photocrosslinkable chitosan as a dressing for wound occlusion and accelerator in healing process. Biomaterials 23, 833-840 (2002).

12. Cheung, R. C. F., Ng, T. B., Wong, J. H. \& Chan, W. Y. Chitosan: an update on potential biomedical and pharmaceutical applications. Mar. Drugs 13, 5156-5186 (2015).

13. Hu, L. et al. pH-sensitive carboxymethyl chitosan hydrogels via acid-labile ortho ester linkage for potential biomedical applications. Carbohydr. Polym. 178, 166-179 (2017).

14. Qiao, X. et al. Evaluation of a photocrosslinkable hydroxyethyl chitosan hydrogel as a potential drug release system for glaucoma surgery. J. Mater. Sci. 28, 149 (2017).

15. Yang, B. et al. Facilely prepared inexpensive and biocompatible self-healing hydrogel: a new injectable cell therapy carrier. Polym. Chem. 23, 3235-3238 (2012).

16. Tseng, T. C. et al. An injectable, self-healing hydrogel to repair the central nervous system. Adv. Mater. 27, 3518-3524 (2015).

17. Tseng, T. C., Hsieh, F. Y., Theato, P., Wei, Y. \& Hsu, S. H. Glucose-sensitive selfhealing hydrogel as sacrificial materials to fabricate vascularized constructs. Biomaterials 133, 20-28 (2017).

18. Hsieh, F. Y., Tao, L., Wei, Y. \& Hsu, S. H. A novel biodegradable self-healing hydrogel to induce blood capillary formation. NPG Asia Mater. 9, e363 (2017).

19. Cheng, Q., Wang, S. Q., Rials, T. G. \& Lee, S. H. Physical and mechanical properties of polyvinyl alcohol and polypropylene composite materials reinforced with fibril aggregates isolated from regenerated cellulose fibers. $\mathrm{Cel}$ lulose 14, 593-602 (2017).

20. Wang, S. Q. \& Cheng, Q. Z. A novel process to isolate fibrils from cellulose fibers by high-intensity ultrasonication, part 1: process optimization. J. Appl. Polym. Sci. 113, 1270-1275 (2009).

21. Jarvis, M. Chemistry - cellulose stacks up. Nature 426, 611-612 (2003).

22. Hepworth, D. G. \& Bruce, D. M. The mechanical properties of a composite manufactured from non-fibrous vegetable tissue and PVA. Compos. Part A Appl. Sci. Manuf. 31, 283-285 (2000).

23. Saito, T., Kimura, S., Nishiyama, Y. \& Isogai, A. Cellulose nanofibers prepared by TEMPO-mediated oxidation of native cellulose. Biomacromolecules $\mathbf{8}$, 2485-2491 (2007)

24. Fujisawa, S., Ikeuchi, T., Takeuchi, M., Saito, T. \& Isogai, A. Superior reinforcement effect of TEMPO-oxidized cellulose nanofibrils in polystyrene matrix: optical, thermal, and mechanical studies. Biomacromolecules 13, 2188-2194 (2012).
25. Huang, C. F., Chen, J. K., Tsai, T. Y., Hsieh, Y. A. \& Lin, K. Y. A. Dual-functionalized cellulose nanofibrils prepared through TEMPO-mediated oxidation and surface-initiated ATRP. Polymer 72, 395-405 (2015).

26. Park, M., Lee, D. \& Hyun, J. Nanocellulose-alginate hydrogel for cell encapsulation. Carbohydr. Polym. 116, 223-228 (2015).

27. Sinclair, A. et al. Cellulose nanofibers produced from various agricultural residues and their reinforcement effects in polymer nanocomposites. J. Appl. Polym. Sci. 135, 46304 (2018).

28. Huang, T., Kuboyama, K., Fukuzumi, H. \& Ougizawa, T. PMMA/TEMPOoxidized cellulose nanofiber nanocomposite with improved mechanical properties, high transparency and tunable birefringence. Cellulose $\mathbf{2 5}$, 2393-2403 (2018).

29. Hossen, M. R. et al. Wet stable and mechanically robust cellulose nanofibrils (CNF) based hydrogel. Polymer 151, 231-241 (2018).

30. Zhang, Y., Tao, L., Li, S. \& Wei, Y. Synthesis of multiresponsive and dynamic chitosan-based hydrogels for controlled release of bioactive molecules. Biomacromolecules 12, 2894-2901 (2011).

31. Hsu, Y. C. et al. Brain-specific 1B promoter of FGF1 gene facilitates the isolation of neural stem/progenitor cells with self-renewal and multipotent capacities. Dev. Dyn. 238, 302-314 (2009).

32. Collins, S. J. et al. The prion protein regulates beta-amyloid mediated selfrenewal of neural stem cells in vitro. Stem Cell Res. Ther. 6, 60 (2015).

33. Collins, S. J. \& Haigh, C. L. Simplified murine 3D neuronal cultures for investigating neuronal activity and neurodegeneration. Cell Biochem. Biophys. 75, 3-13 (2017).

34. Wu, C. C. et al. On the crucial cerebellar wound healing-related pathways and their cross-talks after traumatic brain injury in danio rerio. Plos ONE 9, e97902 (2014).

35. Hsieh, F. Y., Lin, H. H. \& Hsu, S. H. 3D bioprinting of neural stem cell-laden thermoresponsive biodegradable polyurethane hydrogel and potential in central nervous system repair. Biomaterials 71, 48-57 (2015).

36. Wang, Y. A. et al. An injectable interpenetrating polymer network hydrogel with tunable mechanical properties and self-healing abilities. Macromol. Chem. Phys. 218, 1-12 (2017).

37. Liu, H. C. et al. Self-healing and injectable polysaccharide hydrogels with tunable mechanical properties. Cellulose 25, 559-571 (2018).

38. Chen, Y. J. et al. Injectable self-healing zwitterionic hydrogels based on dynamic benzoxaborole-sugar interactions with tunable mechanical properties. Biomacromolecules 19, 596-605 (2018).

39. Su, E. \& Okay, O. Hybrid cross-linked poly(2-acrylamido-2-methyl-1-propanesulfonic acid) hydrogels with tunable viscoelastic, mechanical and self-healing properties. React. Funct. Polym. 123, 70-79 (2018).

40. Ye, J. et al. Surface modification of electrospun TPU nanofiber scaffold with CNF particles by ultrasound-assisted technique for tissue engineering. Macromol. Mater. Eng. 302, 1-9 (2017).

41. Qu, J., Zhao, X., Ma, P. X. \& Guo, B. pH-responsive self-healing injectable hydrogel based on $\mathrm{N}$-carboxyethyl chitosan for hepatocellular carcinoma therapy. Acta Biomater. 58, 168-180 (2017).

42. Lü, S. et al. Injectable and self-healing carbohydrate-based hydrogel for cell encapsulation. ACS Appl Mater. Interfaces 7, 13029-13037 (2015).

43. Abu-Danso, E., Srivastava, V., Sillanpaa, M. \& Bhatnagar, A. Pretreatment assisted synthesis and characterization of cellulose nanocrystals and cellulose nanofibers from absorbent cotton. Int. J. Biol. Macromol. 102, 248-257 (2017).

44. Nguyen, T. H. M. et al. In vitro and in vivo acute response towards injectable thermosensitive chitosan/TEMPO-oxidized cellulose nanofiber hydrogel. Carbohydr. Polym. 180, 246-255 (2018).

45. Chen, P. H. et al. A novel polyurethane/cellulose fibrous scaffold for cardiac tissue engineering. RSC Adv. 5, 6932-6939 (2015).

46. Kim, M. \& Kim, G. 3D multi-layered fibrous cellulose structure using an electrohydrodynamic process for tissue engineering. J. Colloid Interface Sci. 457, 180-187 (2015).

47. Gao, B., Qu, J., Zhao, X. \& Zhang, M. Degradable conductive self-healing hydrogels based on dextran-graft-tetraaniline and N-carboxyethyl chitosan as injectable carriers for myoblast cell therapy and muscle regeneration. Acta Biomater. 84, 190-193 (2019).

48. Mckee, J. R. et al. Thermoresponsive nanocellulose hydrogels with tunable mechanical properties. ACS Macro Lett. 3, 266-270 (2014).

49. Markstedt, K. et al. 3D bioprinting human chondrocytes with nanocellulosealginate bioink for cartilage tissue engineering applications. Biomacromolecules 16, 1489-1496 (2015) 
50. Aarstad, O. et al. Mechanical properties of composite hydrogels of alginate and cellulose nanofibrils. Polymers 9, 378 (2017).

51. Yoon, H. Y. et al. Glycol chitosan nanoparticles as specialized cancer therapeutic vehicles: sequential delivery of doxorubicin and Bcl-2 siRNA. Sci. Rep. 4 6878 (2014)

52. Wei, Z. et al. Self-healing polysaccharide-based hydrogels as injectable carriers for neural stem cells. Sci. Rep. 6, 37841 (2016).

53. Dong, R. N., Zhao, X., Guo, B. L. \& Ma, P. X. Self-healing conductive injectable hydrogels with antibacterial activity as cell delivery carrier for cardiac cell therapy. Acs Appl. Mater. Interfaces 8, 17138-17150 (2016).

54. Winkle, J. J. et al. Modeling mechanical interactions in growing populations of rod-shaped bacteria. Phys. Biol. 14, e55001 (2017).

55. Cox, B. N. \& Landis, C. M. Solitary waves in morphogenesis: determination fronts as strain-cued strain transformations among automatous cells. J. Mech. Phys. Solids 111, 239-276 (2018).

56. Panchision, D. M. The role of oxygen in regulating neural stem cells in development and disease. J. Cell Physiol. 220, 562-568 (2009).

57. Xavier, J. M., Rodrigues, C. M. P. \& Sola, S. Mitochondria: major regulators of neural development. Neuroscientist 22, 346-358 (2016).

58. Qu, J. et al. Antibacterial adhesive injectable hydrogels with rapid self-healing, extensibility and compressibility as wound dressing for joints skin wound healing. Biomaterials 183, 185-199 (2018).
59. Waters, R. et al. Stem cell-inspired secretome-rich injectable hydrogel to repair injured cardiac tissue. Acta Biomater. 69, 95-106 (2018).

60. Luan, C. X., Liu, P., Chen, R. Z. \& Chen, B. A. Hydrogel based 3D carriers in the application of stem cell therapy by direct injection. Nanotechnol. Rev. $\mathbf{6}$, 435-448 (2017)

61. Wu, Y., Wang, L., Gao, B. \& Ma, P. X. Interwoven aligned conductive nanofiber yarn/hydrogel composite scaffolds for engineered 3D cardiac anisotropy. ACS Nano. 11, 5646-5659 (2017)

62. Lu, W. N. et al. Functional improvement of infarcted heart by co-Injection of embryonic stem cells with temperature-responsive chitosan hydrogel. Tissue Eng. Part A 15, 1437-1447 (2009)

63. Johnson, T. D. \& Christman, K. L. Injectable hydrogel therapies and their delivery strategies for treating myocardial infarction. Expert Opin. Drug Deliv. 10 59-72 (2013).

64. Cheng, T. Y. et al. Neural stem cells encapsulated in a functionalized selfassembling peptide hydrogel for brain tissue engineering. Biomaterials $\mathbf{3 4}$, 2005-2016 (2013)

65. Unterman, S. et al. Hydrogel nanocomposites with independently tunable rheology and mechanics. ACS Nano. 11, 2598-2610 (2017).

66. Alam, A. et al. Polymer composite hydrogels containing carbon nanomaterials-morphology and mechanical and functional performance. Prog. Polym. Sci. 77, 1-18 (2018). 